\title{
Differential secretion of Dictyostelium discoideum proteinases
}

\author{
Michael J. North, ${ }^{*} \dagger$ Karl J. Franek and David A. Cotter \\ Department of Biological Sciences, University of Windsor, Windsor, Ontario N9B 3P4, Canada
}

(Received 12 September 1989; revised 2 January 1990; accepted 25 January 1990)

\begin{abstract}
Proteinases secreted by Dictyostelium discoideum myxamoebae during growth and starvation were analysed using three arginine-containing peptide nitroanilides as substrates. The enzymes were secreted differentially, both during growth and after resuspension of the myxamoebae in phosphate buffer. Secretion was stimulated by the addition of sucrose to starvation buffer, but this did not affect the type of proteinase released. Activity towards $\boldsymbol{N}$-benzoyl-Lprolyl-L-phenylalanyl-L-arginine 4-nitroanilide (BzPFRase activity) was released in large quantities, and over $80 \%$ of the total activity in starved suspensions was extracellular within $6 \mathrm{~h}$ of transfer to buffer containing sucrose. A smaller fraction of the total activity towards $N$-carbobenzoxy-L-arginyl-L-arginine 4-nitroanilide (ZRRase activity) was secreted. Extracellular activity towards $\boldsymbol{N}$-carbobenzoxy-L-tyrosyl-L-lysyl-L-arginine 4nitroanilide (ZYKRase) was either too low to be detected or present in quantities which were small enough to be accounted for by cell lysis. BzPFRase and ZRRase activities were both inactivated by E-64 and two peptidyldiazomethanes, specific inhibitors of cysteine proteinases. ZYKRase activity was also inhibited by peptidyldiazomethanes but E-64 was effective only at high concentrations. When electrophoresis in polyacrylamide gels containing gelatin (gelatin-SDS-PAGE) was used to analyse the proteinases involved, one cysteine proteinase, ddCP42, correlated exactly with the secreted BzPFRase activity under all conditions, although contributions to this activity from other proteinases are also likely. Overall, the results demonstrate significant differences between individual proteinases with respect to secretion which suggests that their physiological roles may differ.
\end{abstract}

\section{Introduction}

The cellular slime mould Dictyostelium discoideum produces a number of different proteinases of which the cysteine proteinases have proved to be of greatest interest (North, 1985, 1988; North et al., 1988, 1990). Multiple forms of cysteine proteinases are apparent, and these are subject to both nutritional and developmental control. This multiplicity raises the question as to whether the physiological roles of individual proteinases differ. If so, their location inside and outside the cell may also be expected to differ.

Many of the acid hydrolases of $D$. discoideum are believed to be lysosomal. In fact, the organism provides

† Permanent address: Department of Biological and Molecular Sciences, School of Natural Sciences, University of Stirling, Stirling FK9 4LA, UK.

Abbreviations: $\mathrm{Bz}, \mathrm{N}$-benzoyl; $\mathrm{Z}, \mathrm{N}$-benzyloxycarbonyl; Nan, 4nitroaniline; BzPFRase, Bz-Pro-Phe-Arg-Nan hydrolysing activity; ZRRase, Z-Arg-Arg-Nan hydrolysing activity; ZYKRase, Z-Tyr-LysArg-Nan hydrolysing activity; $\mathrm{CHN}_{2}$, diazomethane; E-64, L-3carboxy-2,3-trans-epoxypropionyl-leucylamido-(4-guanidino)butane. an excellent system for studies of lysosomal enzymes since the enzymes are present in abundance, and the availability of mutants which are defective at various stages of maturation has allowed the elucidation of details of their synthesis, modification, processing and secretion (see Cardelli et al., 1987). A number of these enzymes are secreted both during growth in axenic culture and when cells are starved. There are, however, significant differences between individual hydrolases with respect to their secretion kinetics, and it has been suggested that this could be due to their differential location within subpopulations of lysosomes (Dimond et al., 1981). It was, therefore, of interest to investigate whether cysteine proteinases are differentially secreted. In order to distinguish between different enzymes two approaches have been employed. These involve assays using chromogenic peptides and electrophoresis in substrate-containing gels. The use of the chromogenic substrates showed that very different enzymes can be detected even with closely related substrates, and both approaches have revealed differential proteinase secretion. 


\section{Methods}

Organism, growth and sample preparation. Dictyostelium discoideum strain AX2 (ATCC 24397) was obtained from Dr J. R. Williams (Imperial Cancer Research Fund, South Mimms, Herts, UK). Myxamoebae were grown at $23^{\circ} \mathrm{C}$ on a gyratory shaker in HL5 medium containing glucose (Watts \& Ashworth, 1970).

For studies of secretion, the cells were harvested during the midexponential phase by centrifugation $(500 \mathrm{~g}, 5 \mathrm{~min})$, washed twice with sterile buffer (10 mM-potassium phosphate buffer, $\mathrm{pH} \mathrm{6.5)}$ and resuspended in $20 \mathrm{ml}$ of buffer in $100 \mathrm{ml}$ Erlenmeyer flasks. With some suspensions $0.1 \mathrm{M}$-sucrose was included in the buffer. The cells were incubated with short reciprocating strokes at $23^{\circ} \mathrm{C}$ and $2 \mathrm{ml}$ samples withdrawn periodically. Cell pellets were obtained by centrifugation $(1000 \mathrm{~g}, 5 \mathrm{~min})$ and frozen immediately. The supernatant was retained and stored at $-15^{\circ} \mathrm{C}$ until required. Immediately prior to proteinase analysis the cell pellets were lysed by adding $10 \mathrm{~mm}$-sodium phosphate buffer, $\mathrm{pH} 6.0$, containing $0.1 \%$ Triton $\mathrm{X}-100$ and vortexing.

Enzyme assays. Proteinase activity was measured using peptide nitroanilides as substrates by a micro-method based on an assay described previously (North et al., 1983). Assays were done in 96-well microtitre plates and the activity followed by using a Biotek microtitre plate reader fitted with a $405 \mathrm{~nm}$ filter. For samples possessing high activity (all cell extracts and some extracellular samples), $15 \mu \mathrm{l}$ was placed in a well and the reaction started by the addition of $0.15 \mathrm{ml}$ buffer containing $0.1 \mathrm{~mm}$ substrate and $1 \mathrm{~mm}$-DTT unless otherwise indicated. For low activity samples (some extracellular samples) the assays were set up by placing $45 \mu \mathrm{l}$ of buffer containing $0.33 \mathrm{~mm}$ substrate and, if required, $3.3 \mathrm{~mm}$-DTT in each well and then adding $0.12 \mathrm{ml}$ sample. In all assays the buffer used was $0.1 \mathrm{M}$-sodium phosphate, $\mathrm{pH} 6 \cdot 0$, and the microtitre plates were incubated at $23^{\circ} \mathrm{C}$. Specific activity was calculated from the initial linear increase in absorbance in units of nmol nitroaniline released $\operatorname{~min}^{-1}$ (mg protein $)^{-1}$. Protein was determined by the method of Bradford (1976) using bovine albumin as standard.

The effect of inhibitors was tested by mixing a solution of inhibitor (prepared in water for E-64 and acetonitrile for the peptidyldiazomethanes) with sample containing proteinase in individual wells, standing for $5 \mathrm{~min}$ and then adding substrate solution as above. When used, the final concentration of acetonitrile in the reaction mix was $5 \%(\mathrm{v} / \mathrm{v})$ which had no effect on any of the activities measured.

Electrophoretic analysis of proteinases. Proteinases were analysed by the gelatin-SDS-PAGE procedure described previously (North et al., 1988) except that a Bio-Rad mini Protean system was used. Because of the smaller size of the gels the following adjustments were made to the method. Samples were loaded in a total volume (including sample buffer) of $20 \mu \mathrm{l}$ containing approximately $10 \mu \mathrm{g}$ protein (cell extracts) or $10 \mu \mathrm{l}$ of medium or buffer. Current was applied at $5 \mathrm{~mA}$ per gel and electrophoresis carried out for 2 to $3 \mathrm{~h}$ until the marker dye had reached the bottom of the separating gel. Gels were incubated in $2.5 \%(\mathrm{v} / \mathrm{v})$ Triton X-100 for $30 \mathrm{~min}$ and in appropriate buffer for $2-4 \mathrm{~h}$ (intracellular samples) or 16-24 $\mathrm{h}$ (extracellular samples) to allow the development of proteinase bands. Unless otherwise indicated, the

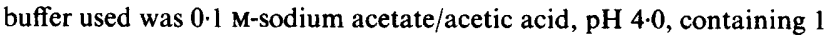
mM-DTT. Proteinase bands were visualized by staining with Coomassie blue and destaining in $10 \%(\mathrm{v} / \mathrm{v})$ acetic acid containing $5 \%(\mathrm{v} / \mathrm{v})$ methanol.

Chemicals. Bz-Pro-Phe-Arg-Nan, gelatin and DTT were from Sigma, Z-Arg-Arg-Nan was obtained from Novabiochem and Z-Tyr-Lys-ArgNan, Z-Lys-Arg-Nan, Z-Phe-Phe-CHN ${ }_{2}$ and Z-Phe-Ala-CHN from Bachem. E-64 was a product of the Peptide Institute and was obtained from Scientific Marketing Associates. All other chemicals were of the highest purity commercially available.

\section{Results}

\section{Intracellular peptide-nitroanilide-hydrolysing activities}

D. discoideum produces enzymes which hydrolyse a number of arginine-containing proteinase substrates (North, 1982a, 1985). The enzymes responsible for most of these activities have been considered to be cysteine proteinases. Many such substrates have now been tested, and of the nitroanilide derivatives, the four which are most rapidly hydrolysed by myxamoebal extracts are BzPro-Phe-Arg-Nan, Z-Arg-Arg-Nan, Z-Tyr-Lys-ArgNan and Z-Lys-Arg-Nan. The first three of these were initially selected for this study solely on the basis that they were rapidly hydrolysed, but their use has also allowed three different types of proteolytic enzymes to be identified. The substrates were hydrolysed by extracts prepared from vegetative myxamoebae at relatively similar rates, with specific activities of $7 \cdot 1 \pm 2 \cdot 1,2 \cdot 9 \pm$ 1.0 and $2.8 \pm 1.9 \mathrm{nmol} \mathrm{min}^{-1}$ (mg protein) ${ }^{-1}$ for Bz-ProPhe-Arg-Nan, Z-Arg-Arg-Nan and Z-Tyr-Lys-Arg-Nan respectively (values are the means of eight determinations and are given $\pm \mathrm{SD}$ ). The Bz-Pro-Phe-Arg-Nan and the Z-Arg-Arg-Nan hydrolysing activities (referred to as BzPFRase and ZRRase respectively) were both stimulated up to 3-fold by DTT at concentrations above $0.1 \mathrm{~mm}$, whereas the activity towards Z-Tyr-Lys-ArgNan (ZYKRase) was unaffected by 1 mM-DTT. BzPFRase and ZRRase activities were both blocked by low concentrations of E-64 (Fig. 1), a specific inhibitor of cysteine proteinases (Kirschke \& Barrett, 1987), ZRRase being the more sensitive. A difference between BzPFRase and ZRRase was apparent when the peptidyldiazomethanes were tested. Z-Phe-Phe- $\mathrm{CHN}_{2}$ severely inhibited BzPFRase but was less effective on ZRRase, whereas Z-Phe-Ala-CHN ${ }_{2}$ inactivated both to a similar degree. E-64 inhibited ZYKRase only slightly at high concentrations. Both peptidyldiazomethanes had an effect on ZYKRase, although here too the inhibition was less than with the other two activities. These results indicate that both BzPFRase and ZRRase must be due to cysteine proteinases. The limited effect of E-64 on ZYKRase and its lack of dependence on DTT suggest, however, that the enzyme(s) responsible for this activity may be of a different catalytic type, although inhibition by the two peptidyldiazomethanes used here is a characteristic of cysteine proteinases.

\section{Extracellular peptide-nitroanilide-hydrolysing activities}

In samples of growth medium, the relative rates of peptide nitroanilide hydrolysis differed significantly from those measured in cell extracts. BzPFRase levels were variable but were always high, and the extracellular 


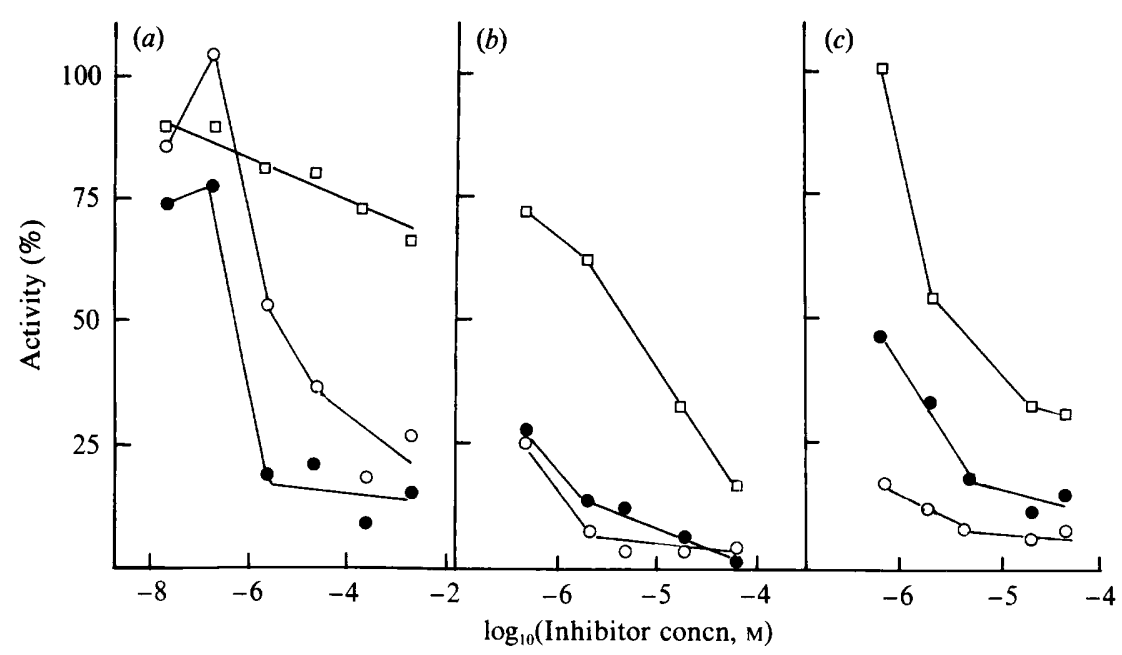

Fig. 1. Inhibition of peptide-nitroanilide-hydrolysing activities. Assays were done with extracts prepared from vegetative myxamoebae treated with inhibitors as described in Methods. Inhibitor concentrations given are those in the final assay mix. During preincubation these were 10-fold higher. The activity is given as a percentage of that measured in the absence of inhibitor. (a) E-64; (b) Z-Phe-Ala$\mathrm{CHN}_{2}$; (c) Z-Phe-Phe-CHN ${ }_{2}$. O, BzPFRase; O, ZRRase; $\square$, ZYKRase.

activity accounted for up to $45 \%$ of the total (intracellular plus extracellular) activity in late-exponential phase cultures. Extracellular ZRRase was also present but at a lower level, with a maximum of $30 \%$ of the total activity present in the medium. In contrast, ZYKRase was absent from the growth medium. The sensitivity of the assay method was such that ZYKRase activity at a level 50-fold less than the extracellular BzPFRase would have been detected had it been present. The lack of ZYKRase activity was not due to its inhibition nor to its inactivation by components of the medium. ZYKRase was detectable in extracts of cells lysed in growth medium (fresh or spent) and this activity was retained even after $24 \mathrm{~h}$ at $22^{\circ} \mathrm{C}$.

During starvation in shaken buffered suspensions, axenically grown myxamoebae release a number of acid hydrolases (Dimond et al., 1981). This process can be stimulated by sucrose and other disaccharides (Crean \& Rossomando, 1979; Seshadri et al., 1986). The relative levels of the secreted peptide-nitroanilide-hydrolysing enzymes in starvation buffer were similar to those found in growth medium (Fig. 2). The cells released large quantities of BzPFRase and a smaller quantity of ZRRase, but no extracellular ZYKRase was detected. Sucrose stimulated the release but did not affect the type of activity secreted. When sucrose was present, over $80 \%$ of the total BzPFRase activity in the suspension was extracellular after $6 \mathrm{~h}$. Its appearance in the buffer was accompanied by an equivalent fall in intracellular activity. For ZRRase, approximately $35 \%$ of the total activity was extracellular $6 \mathrm{~h}$ after the cells were resuspended in sucrose-containing buffer. Only a small proportion of the ZYKRase activity was released under these conditions, but this was so low $(\sim 10 \%$ of the total in the suspension after $6 \mathrm{~h}$ ) that it could have resulted from cell lysis (Dimond et al., 1981). The results shown in Fig. 2 are those for one of a number of independent experiments. Although the actual secretion kinetics differed slightly between the experiments, the relative levels of the three activities and the degree of stimulation by sucrose were the same.

To gain more information on the specificity of the enzymes involved, activity towards Z-Lys-Arg-Nan was also measured. This substrate has the same pair of basic amino acids at the $P_{1}$ and $P_{2}$ positions (as defined by Schechter \& Berger, 1967) as Z-Tyr-Lys-Arg-Nan, but like Z-Arg-Arg-Nan has a benzyloxycarbonyl group rather than an amino acid residue in the $P_{3}$ position and no group in the $\mathrm{P}_{4}$ position. Activity towards Z-Lys-ArgNan was secreted, and in all respects, including activation by DTT and inactivation by cysteine proteinase inhibitors, it was similar to ZRRase and not to ZYKRase (results not shown).

\section{Recovery of enzyme activity}

Because the extent of the depletion of BzPFRase from sucrose-treated cells was so large it was of interest to establish how rapidly the cells could recover intracellular activity once the stimulus for secretion had been removed. The ability of starved myxamoebae to reaccumulate hydrolases had not previously been investigated. When cells starved in buffer for $6 \mathrm{~h}$ were returned to growth medium, there was a small increase in the intracellular BzPFRase activity (Fig. $3 a$ ). The rate of enzyme accumulation was much more rapid in cells 


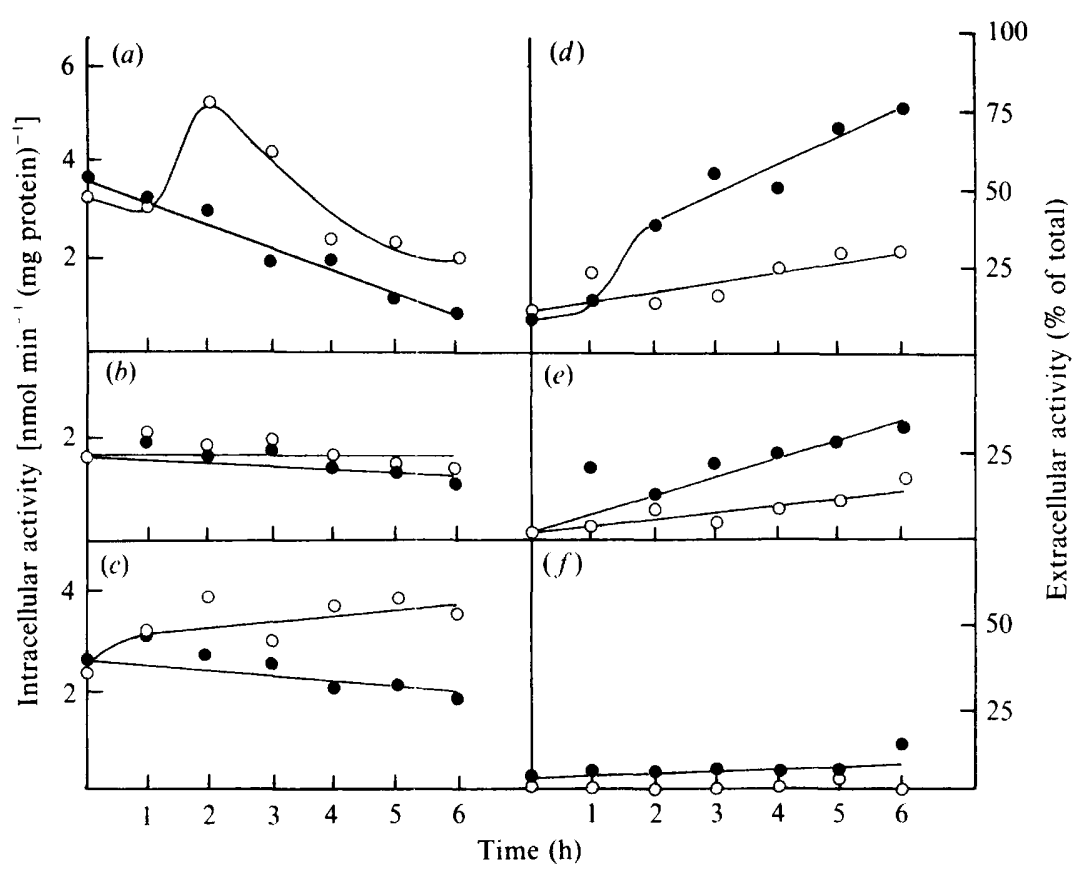

Fig. 2. Release of enzyme activity during starvation. Washed myxamoebae were resuspended at $1.5 \times 10^{6}$ cells ml $^{-1}$ in phosphate buffer with $(O)$ or without $(O) 0 \cdot 1 \mathrm{M}$-sucrose. $(a-c)$ Intracellular activity (specific activity); $(d-f)$ extracellular activity (given as a percentage of the total activity present in buffer and cells). $(a, d)$ BzPFRase; $(b, e)$ ZRRase; $(c, f)$ ZYKRase. The levels of extracellular activity after 6 $\mathrm{h}$ in the control suspensions were 0.70, 0.23 and $0 \mathrm{nmol} \mathrm{min}^{-1}$ (mg cell protein $)^{-1}$ for BzPFRase, ZRRase and ZYKRase respectively; the equivalent levels for the sucrose-treated suspensions were $1.96,0.43$ and $0.33 \mathrm{nmol} \mathrm{m^{-1 }}\left(\mathrm{mg}^{2} \text { cell protein }\right)^{-1} .10^{7}$ cells contain about $1 \mathrm{mg}$ protein.

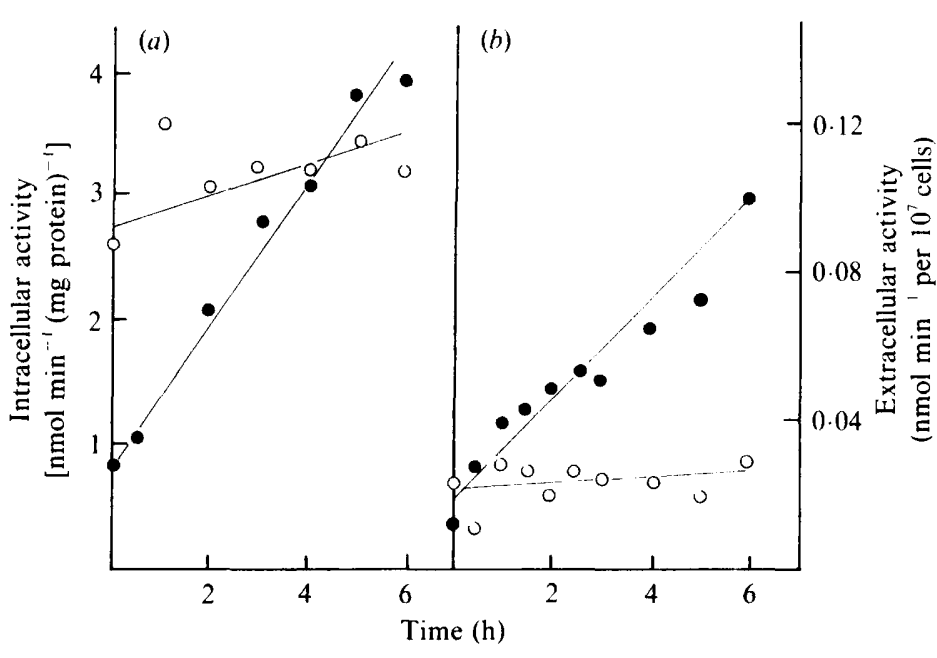

Fig. 3. Recovery of BzPFRase activity by cells starved in buffer. Washed myxamoebae were resuspended at $1.25 \times 10^{7}{\text { cells } \mathrm{ml}^{-1}}^{-1}$ in phosphate buffer with $(O)$ and without $(O) 0.1 \mathrm{M}$-sucrose and shaken for $6 \mathrm{~h}$. The cells were then harvested, washed and resuspended in an equal volume of growth medium. (a) Intracellular activity and $(b)$ extracellular activity in the cultures after restoration to growth medium. which had been treated with sucrose and had consequently lost more BzPFRase. The sucrose-treated cells also continued to secrete large quantities of BzPFRase into growth medium (Fig. $3 b$ ), in contrast to the cells starved in sucrose-free buffer which produced little extracellular activity. The stimulatory effect of sucrose on secretion persisted, therefore, after it had been removed from the cells.

\section{Gelatin-SDS-PAGE analysis}

Enzymes which might contribute to the peptide-nitroanilide-hydrolysing activities were investigated using gelatin-SDS-PAGE. Electrophoretic separation of $D$. discoideum proteinases in gelatin-containing polyacrylamide gels reveals multiple cysteine proteinases, and some differences between the intracellular proteinases 

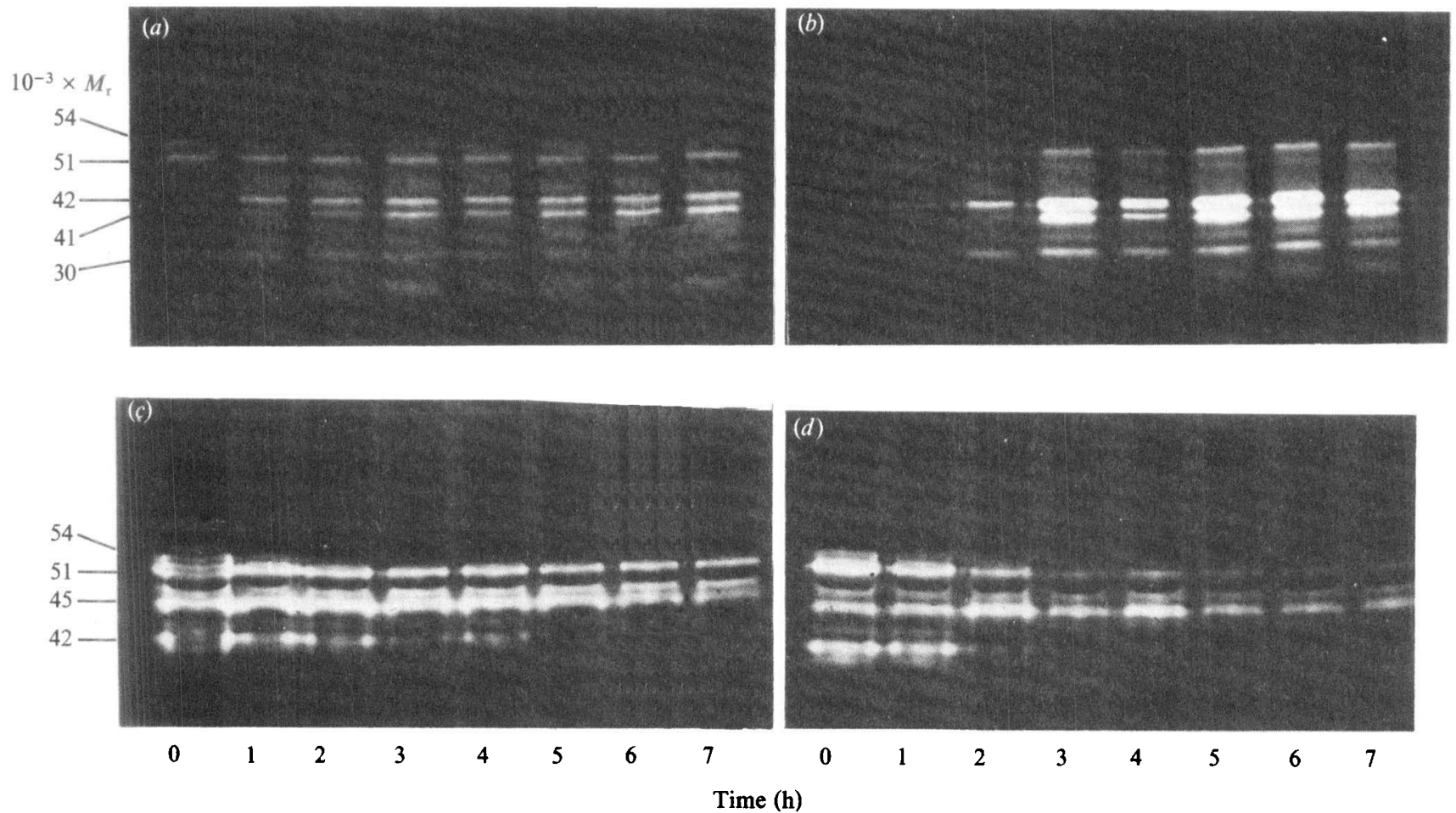

Fig. 4. Release of proteinases into buffer during starvation. Washed myxamoebae were resuspended at $1.0 \times 10^{7}$ cells $\mathrm{ml}^{-1}$ in phosphate buffer alone $(a, c)$ or phosphate buffer containing $0 \cdot 1 \mathrm{M}$-sucrose $(b, d) .(a, b)$ Extracellular samples; $(c, d)$ cell samples. The apparent $M_{\mathrm{r}}$ values of the proteinases are indicated.

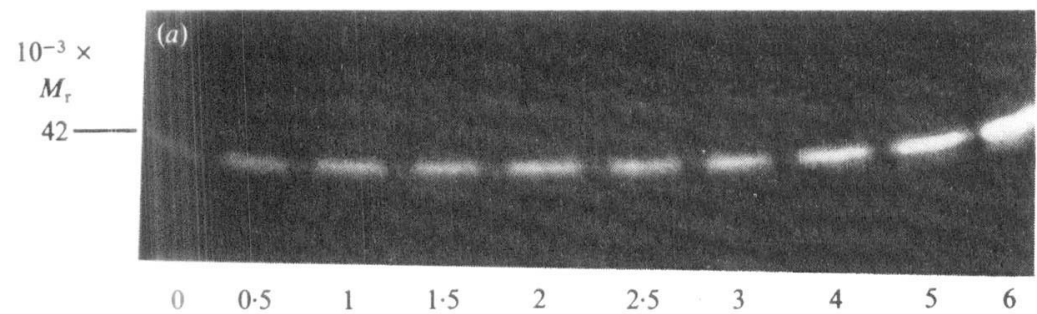

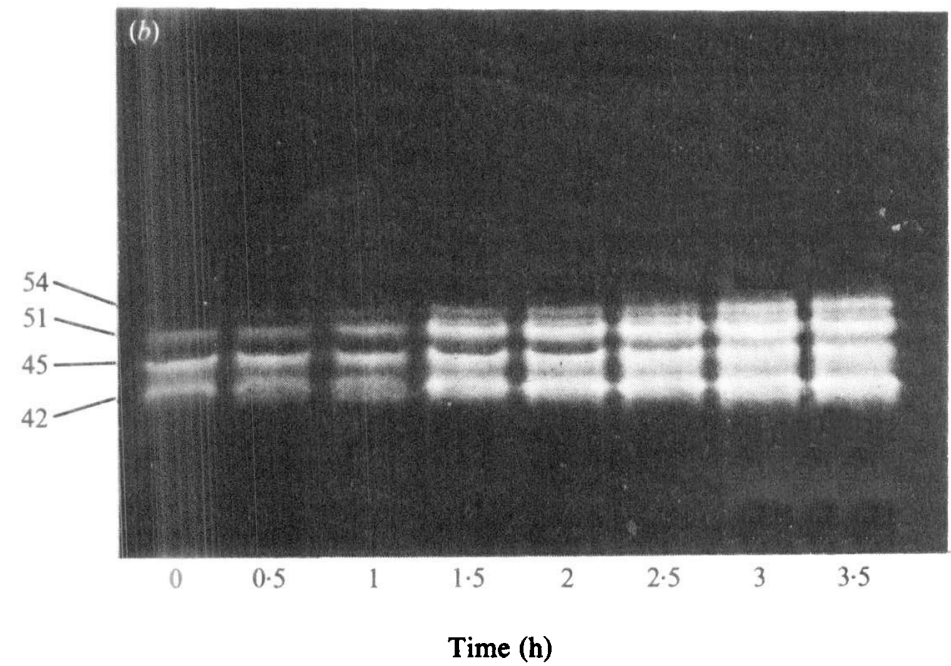

Fig. 5. Recovery of proteinase activity after restoration of starved cells to growth medium. Washed myxamoebae were resuspended at $1.0 \times 10^{7}$ cells $\mathrm{ml}^{-1}$ in phosphate buffer containing $0 \cdot 1 \mathrm{M}$-sucrose and incubated for $6 \mathrm{~h}$. The cells were then collected by centrifugation and resuspended in growth medium at the same density. (a) Extracellular and $(b)$ intracellular proteinases in the culture after restoration to growth medium. The apparent $M_{\mathrm{r}}$ values of the proteinases are indicated. 
and extracellular proteinases have already been described (North et al., 1988). The present study confirmed these differences. The behaviour of one proteinase in particular, ddCP42, paralleled that of the BzPFRase activity. ddCP42 (Dictyostelium discoideum cysteine proteinase, $M_{\mathrm{r}} \underline{42000)}$ is found in the growth medium (North et al., 1988) and was also released in significant quantities during starvation in phosphate buffer (Fig. 4). A second proteinase, ddCP41, usually accompanied ddCP42 but was often of lower activity. Secretion of other enzymes, including ddCP51 and ddCP30, was also observed. When secretion was stimulated by sucrose, ddCP42 and ddCP41 were released more rapidly. Release of ddCP42 was accompanied by a corresponding depletion of the intracellular activity, which occurred more rapidly when sucrose was present (Fig. 4). The intracellular levels of some other enzymes, including ddCP51, also decreased but less rapidly than ddCP42. Some cysteine proteinases, notably ddCP45, were retained by the cells. After being restored to growth medium, sucrose-treated cells continued to secrete ddCP42 which accumulated in the medium during the next $6 \mathrm{~h}$ (Fig. $5 a$ ). The enzyme also reappeared in the cells (Fig. $5 b$ ). Secretion of other extracellular proteinases were much less apparent than during starvation in buffer (Fig. 4). Cells previously starved in buffer alone secreted very little proteinase and, in fact, released ddCP51 rather than ddCP42 (results not shown).

\section{Discussion}

The results presented here extend those of an earlier study in which secretion of proteinases by $D$. discoideum was examined (North, 1982b). Previously, no major differences between the intracellular and extracellular proteinases had been observed. Here, however, the methods we have used have allowed the identification of three types of proteolytic activity which were found to be differentially secreted.

The first of the activities was BzPFRase which was secreted in large quantities and found at high levels in both growth medium and starvation buffer. The secretion of this proteolytic activity was, like that of other acid hydrolases (Seshadri et al., 1986), stimulated by sucrose. ddCP42, one of several Dictyostelium cysteine proteinases detected by gelatin-SDS-PAGE, behaved exactly like the BzPFRase and may be responsible for a significant proportion of the total BzPFRase activity. Some contribution from other enzymes including ddCP41 and ddCP30 is also likely, however. This is especially so for ddCP30 (previously called proteinase B), a cysteine proteinase which had already been purified from myxamoebal extracts and shown to hydrolyse Bz-
Pro-Phe-Arg-Nan (North \& Whyte, 1984). ddCP30 does not show up well on gelatin-SDS-PAGE gels (North $e t$ al., 1988), presumably because it is not as active on gelatin as are the larger proteinases. Some secreted ddCP30 activity was apparent, however (Fig. 3).

These results have shown for the first time that cells depleted of a secreted enzyme during starvation in sucrose-containing buffer can accumulate it again quite quickly when they are restored to growth medium. This was seen with both the BzPFRase activity and the ddCP42 proteinase band. It seems likely that starvation had two effects on the level of intracellular activity, firstly, stimulating secretion, and secondly, stopping any further synthesis of the enzyme(s). Restoration of the cells to growth medium allowed synthesis to restart, but, if they had been treated with sucrose during starvation, the cells did not immediately reduce their secretion rate. This secretion/recovery procedure may prove very useful in future studies concerned with the biosynthesis, processing and targeting of secreted enzymes as these processes may now be analysed in the absence of large pre-existing pools of active enzyme.

Although the second activity, ZRRase, showed some similarities to BzPFRase, the enzymes involved were not identical. The activity was secreted, but to a more limited extent than BzPFRase. Like BzPFRase, ZRRase was inhibited by cysteine proteinase inhibitors, but there were differences: in particular, Z-Phe-Phe- $\mathrm{CHN}_{2}$ was less effective. Furthermore, some separation of the two activities can be achieved by ion-exchange chromatography and gel filtration (M. J. North, unpublished observations).

On the basis of their sensitivity to inhibitors, BzPFRase and ZRRase can both be considered to be cysteine proteinase activities, and a comparison with the lysosomal cysteine proteinases of mammalian cells is therefore relevant. Lysosomal cathepsin L prefers substrates with hydrophobic residues at the $P_{2}$ position and is very active on peptide substrates with Phe-Arg at the $P_{2}-P_{1}$ positions. The latter are also hydrolysed by cathepsin B, which, in contrast to cathepsin $\mathrm{L}$, is also active towards substrates with Arg-Arg at the $P_{2}-P_{1}$ positions (Kirschke \& Barrett, 1987; Barrett et al., 1988). The more readily secreted Dictyostelium enzyme(s) with BzPFRase activity may thus resemble cathepsin L and those with ZRRase activity cathepsin B. This suggestion is supported at least in part by the differential effect of $Z$ Phe-Phe- $\mathrm{CHN}_{2}$.

An important feature of the Dictyostelium cysteine proteinases is that they are apparently larger than the mature forms of cathepsin L and cathepsin B. Larger precursors of both of these mammalian cathepsins are synthesized (see Mason et al., 1989), but these are either non-reactive or have limited specificity. The precursor 
forms sometimes possess latent activity, however, and may be activated in vitro, e.g. at low $\mathrm{pH}$. Unlike the mature cathepsins, but in common with the Dictyostelium enzymes, the mammalian cysteine proteinase precursors are alkali tolerant. It is tempting to speculate, therefore, that the cellular slime mould enzymes represent the equivalents of the cathepsin precursors but possess full activity. Only the larger forms of cathepsins $B$ and $L$ are ever found outside the cell (see Barrett $e t$ al., 1988), and there may thus be a link between the ability of the Dictyostelium enzymes to be secreted and their larger size. More detailed characterization is, however, essential before the precise relationships between the cellular slime mould and mammalian enzymes are established.

ZYKRase represents a novel activity in $D$. discoideum. Although the substrate has a pair of basic amino acids, the tyrosine residue in the $P_{3}$ position must prevent it from being hydrolysed by the ZRRase enzyme(s). However, the presence of the tyrosine allowed the substrate to be hydrolysed by an entirely different non-secreted enzyme(s). Despite being identified in a study which had originally focussed on cysteine proteinases, the enzyme(s) involved may not belong to the same family as the secreted cysteine proteinases. The ZYKRase activity was only slightly inhibited by E-64 and was not activated by DTT. In yeast, Z-Tyr-Lys-Arg-Nan and similar peptide derivatives are substrates for proteinase yscF, the product of $K E X 2$ gene which is responsible for processing the precursor protein of the sex pheromone $\alpha$-factor (Wagner et al., 1987; Mizuno et al., 1989). Proteinase yscF is slightly sensitive to E-64 (Wagner et al., 1987), but sequence data have shown that it is a serine proteinase related to subtilisin (Mizuno et al., 1988).

Unlike BzPFRase and ZRRase, which are recovered in particulate fractions, ZYKRase is apparently cytosolic (M. J. North, unpublished observations) and the respective subcellular locations are almost certainly linked to the secretion behaviour. Thus individual proteinases probably encounter different substrate proteins and could be involved in separate physiological processes. The availability of assays which allow these enzymes to be distinguished easily should now permit a more detailed investigation to show that they represent functionally distinct proteinases.

M.J.N. wishes to thank the Carnegie Trust for the Universities in Scotland for support. This work was also supported, in part, by grants from the Natural Sciences and Engineering Research Council of Canada and a NATO Collaborative Research Grant. We are grateful to Kay Nicol for technical assistance.

\section{References}

Barrett, A. J., Buttle, D. J. \& Mason, R. W. (1988). Lysosomal cysteine proteinases. ISI Atlas of Science: Biochemistry 1, 256-260.
BRADFORD, M. M. (1976). A rapid and sensitive method for quantitation of microgram quantities of protein utilizing the principle of protein-dye binding. Analytical Biochemistry 72, 248254.

Cardelit, J. A., Golumbeski, G. S., Woychik, N. A., Mierendorf, R. C. \& Dimond, R. L. (1987). Defining the intracellular localization pathways followed by lysosomal enzymes in Dictyostelium discoideum. Methods in Cell Biology 28, 139-155.

Crean, E. V. \& Rossomando, E. F. (1979). Effects of sugars on glycosidase secretion in Dictyostelium discoideum. Journal of General Microbiology 110, 315-322.

Dimond, R. L., Burns, R. A. \& Jordan, K. B. (1981). Secretion of lysosomal enzymes in the cellular slime mold Dictyostelium discoideum. Journal of Biological Chemistry 256, 6565-6572.

KIRSCHKE, H. \& BARRETT, A. J. (1987). Chemistry of lysosomal proteases. In Lysosomes: Their Role in Protein Breakdown, pp. 193238. Edited by H. Glaumann \& F. J. Ballard. London: Academic Press.

Mason, R. W., Wilcox, D., Wikstrom, P. \& Shaw, E. N. (1989). The identification of active forms of cysteine proteinases in Kirstenvirus-transformed mouse fibroblasts by use of a specific radiolabelled inhibitor. Biochemical Journal 257, 125-129.

Mizuno, K., Nakamura, T., Ohshima, T., Tanaka, S. \& Matsuo, H. (1988). Yeast $K E X 2$ gene encodes an endopeptidase homologous to subtilisin-like serine proteases. Biochemical and Biophysical Research Communications 156, 246-254.

Mizuno, K., Nakamura, T., Ohshima, T., Tanaka, S. \& Matsuo, H. (1989). Characterization of $K E X 2$-encoded endopeptidase from yeast Saccharomyces cerevisiae. Biochemical and Biophysical Research Communications 159, 305-311.

NORTH, M. J. (1982a). Proteolytic activities in Dictyostelium discoideum detected with chromogenic peptide substrates. Experimental Mycology 6, 345-352.

NoRTH, M. J. (1982b). A study of the proteinase activity released by Dictyostelium discoideum during starvation. Journal of General Microbiology 128, 1653-1659.

NORTH, M. J. (1985). Cysteine proteinases of cellular slime moulds. Biochemical Society Transactions 13, 288-290.

NORTH, M. J. (1988). A bacterial factor induces changes in cysteine proteinase forms in the cellular slime mould Dictyostelium discoideum. Biochemical Journal 254, 269-275.

NORTH, M. J. \& Whyte, A. (1984). Purification and characterization of two acid proteinases from Dictyostelium discoideum. Journal of General Microbiology 130, 123-134.

NoRTH, M. J., CoOmBS, G. H. \& BARRY, J. D. (1983). A comparative study of the proteolytic enzymes of Trypanosoma brucei, $T$. equiperdum, T. evansi, T. vivax, Leishmania tarentolae and Crithidia fasciculata. Molecular and Biochemical Parasitology 9, 161-180.

North, M. J., SCOTT, K. I. \& Lockwood, B. C. (1988). Multiple cysteine proteinase forms during the life cycle of Dictyostelium discoideum revealed by electrophoretic analysis. Biochemical Journal 254, 261-268.

North, M. J., Cotter, D. A. \& FraneK, K. J. (1990). Dictyostelium discoideum spore germination: increases in proteinase activity are not directly coupled to the emergence of myxamoebae. Journal of General Microbiology 136, 000-000.

SCHECHTER, I. \& BERGER, A. (1967). On the size of the active site in proteases. I. Papain. Biochemical and Biophysical Research Communications 27, 157-162.

Seshadri, J., Cotter, D. A. \& Dimond, R. L. (1986). The characterization and secretion pattern of the lysosomal trehalases of Dictyostelium discoideum. Experimental Mycology 10, 131-143.

W AGNER, J.-C., EsCHER, C. \& Wolf, D. H. (1987). Some characteristics of hormone (pheromone) processing enzymes in yeast. FEBS Letters 218, 31-34.

WATTS, D. J. \& ASHWORTH, J. M. (1970). Growth of myxamoebae of the cellular slime mould Dictyostelium discoideum in axenic culture. Biochemical Journal 119, 171-174. 